\title{
Emphysematous pyelonephritis associated with calcium oxalate uroliths detected on computed tomography in an awake dog
}

\author{
Ho-Hyun Kwak ${ }^{1}$, Kamal Hany Hussein ${ }^{1,2}$, Heung-Myong Woo ${ }^{1}$, Kyung-Mee Park ${ }^{3, *}$ \\ ${ }^{1}$ College of Veterinary Medicine, Kangwon National University, Chuncheon 24341, Korea \\ ${ }^{2}$ College of Veterinary Medicine, Assiut University, Assiut 71515, Egypt \\ ${ }^{3}$ College of Veterinary Medicine, Chungbuk National University, Cheongju 28644, Korea
}

\begin{abstract}
An 11-year-old female mixed-breed dog admitted due to anorexia and vomiting. Radiography showed 7.6-mm uroliths in the right proximal ureter and an enlarged kidney. Type 1 emphysematous pyelonephritis (EPN) was diagnosed using computed tomography (CT) in the awake dog. Right ureteronephrectomy was performed, and Escherichia coli was isolated. The stone was consisted of $80 \%$ calcium oxalate. At the 6-month follow-up, no complications were observed. This is the first report of EPN caused by calcium oxalate in a dog. In addition, we suggest performing CT in weak animals to diagnose EPN without anesthesia.
\end{abstract}

Keywords: calcium oxalate, dog, Escherichia coli, kidney, pyelonephritis

*Corresponding author

Kyung-Mee Park

College of Veterinary Medicine, Chungbuk National University, 1 Chungdae-ro, Seowongu, Cheongju 28644, Korea

Tel: $+82-43-261-2985$

Fax: $+82-43-267-2595$

E-mail: parkkm@cbnu.ac.kr

ORCID

Ho-Hyun Kwak

https://orcid.org/0000-0002-6773-7686

Kamal Hany Hussein

https://orcid.org/0000-0002-0717-7875

Heung-Myong Woo

https://orcid.org/0000-0003-2105-3913

Kyung-Mee Park

https://orcid.org/0000-0002-7165-6731

Conflict of Interest

The authors declare no conflicts of interest.

Received: January 25, 2020

Accepted: April 24, 2020
Emphysematous pyelonephritis (EPN) is an uncommon destructive gas-producing infection of the renal parenchyma and collecting system [1]. It most commonly occurs as a complication of uncontrolled diabetes mellitus, followed by urinary tract obstruction in $25-40 \%$ of human cases. Escherichia coli is the most common cause, seen in nearly $70 \%$ in human medicine [2]. There are 2 types of EPN in humans. Type 1 EPN is defined as destruction and/or fluid retention in renal parenchyma. Whereas, type 2 is characterized by the fluid accumulation in the renal or perirenal parts and gas production in the collecting system [3]. Type 1 EPN has a poor prognosis compare to type 2. Although EPN has not been classified in the veterinary literature, its cases have been reported, including 2, 1 , and 1 case associated with diabetes, urinary tract infection, and a portosystemic shunt in a dog with high mortality, respectively $[3,4]$. Herein, we report a case of EPN associated with ureteral obstruction by calcium oxalate uroliths in a dog.

An 11-year-old intact female mixed-breed dog was referred because of lethargy, anorexia, and vomiting. On physical examination, no significant abnormality was found. Complete blood count showed mild leukocytosis $(20.75 \times$ $\left.10^{9} / \mathrm{L}\right)$ and thrombocytopenia $\left(134 \times 10^{9} / \mathrm{L}\right)$. Serum chemistry revealed an elevated alkaline phosphate level $(749 \mathrm{U} / \mathrm{L})$. Serum urea nitrogen $(48 \mathrm{mg} / \mathrm{dL})$ and creatinine $(1.6 \mathrm{mg} / \mathrm{dL})$ were unremarkable. The dipstick test revealed proteinuria $(30 \mathrm{mg} / \mathrm{dL})$, slight aciduria $(\mathrm{pH}: 5)$, and normal urinary specific gravity (1.023). Urine cytology revealed rod-shaped bacteria and neutrophils. On radiography, the right kidney was enlarged, with snowman-shaped gas opacity, and 3 radiopaque ureteral uroliths were present in the proximal ureter. The renal pelvis was dilated by echogenic fluid with gas containing a urolith, 7.6 $\mathrm{mm}$ in diameter, with shadowing in the proximal ureter on ultrasonography (Aplio 400; Toshiba Medical Systems Corp., Japan) (Fig. 1). Due to the poor body condition, computed tomography (CT, Alexion Advance; Toshiba Medical Systems Corp.) was performed in the awake dog without anesthesia or sedation in sternal recumbency in a cat restraint bag. In the right kidney, nonenhancing gas material was detected. Three uroliths were found in the right proximal ureter. Based on these results, type 1-like EPN with ureteral uroliths was diagnosed (Fig. 2).

For the right ureteronephrectomy, the renal vessels and proximal ureter 

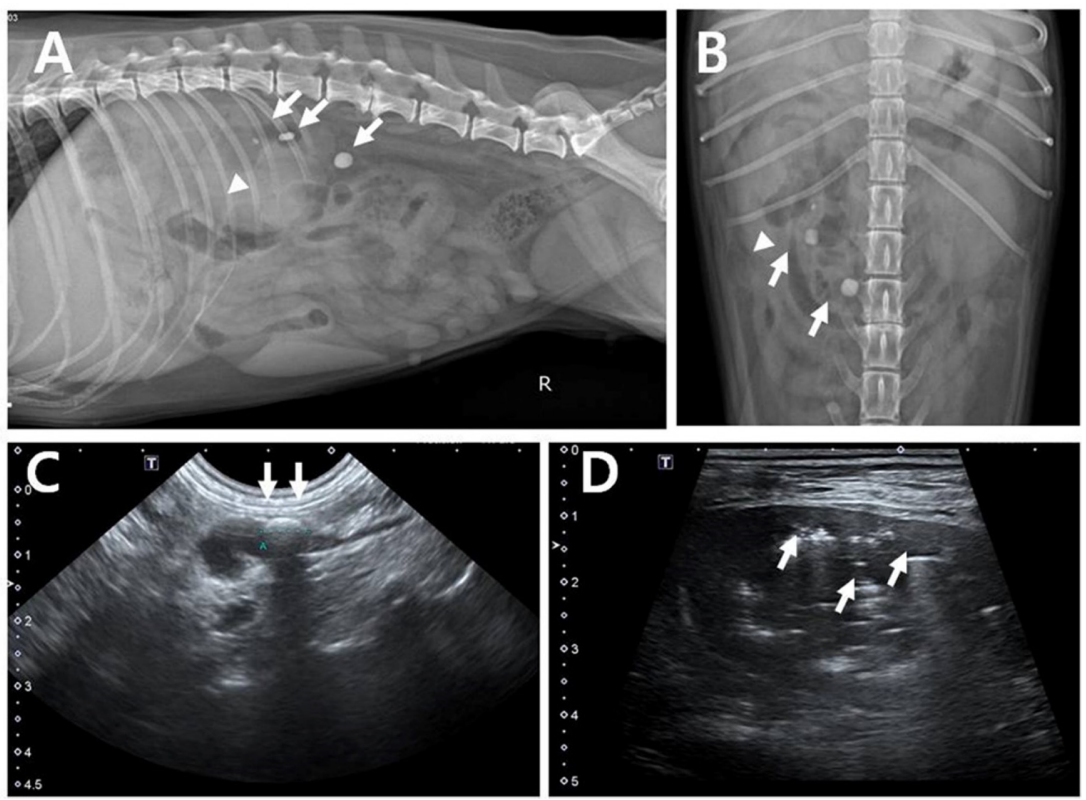

Fig. 1. The radiograph reveals three uroliths (arrows) and snowman-like free gas (arrowhead) in the right proximal ureter (A, B). Ultrasonography reveals free gas in the renal pelvis and dilated proximal ureter with hyperechoic ureteral stones (arrows) (C, D).
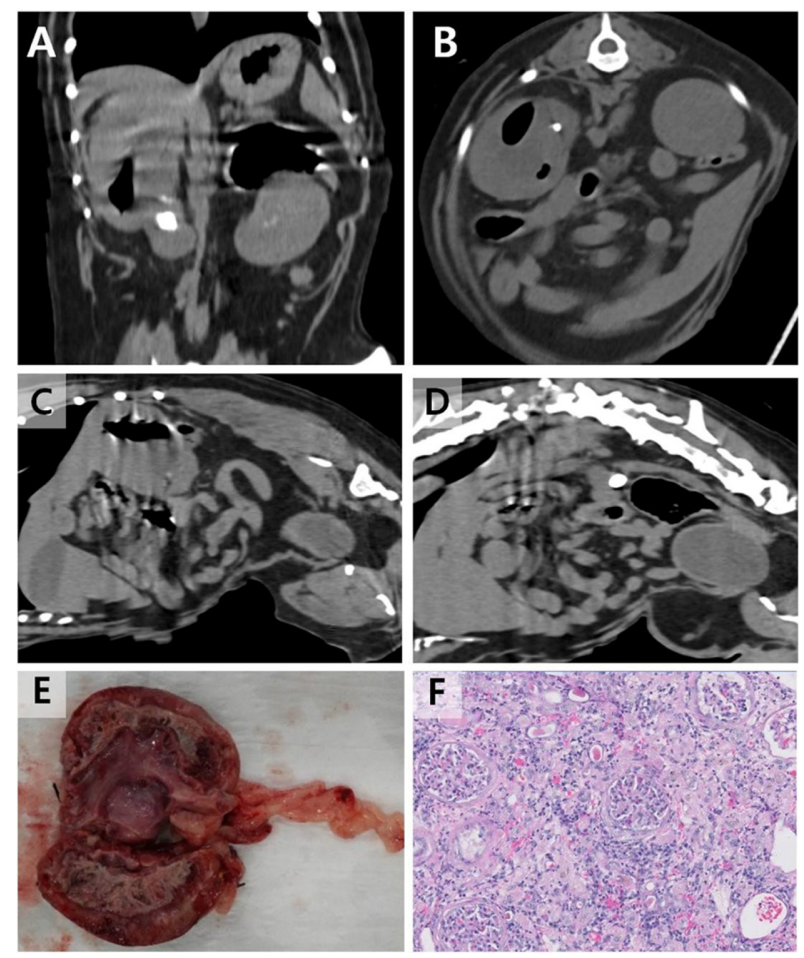

Fig. 2. On abdominal computed tomography, free gas in the right kidney and uroliths in the dilated proximal ureter are seen in the transverse (A, B) and sagittal (C, D) views. Slight motion in the awake patient did not disturb the computed tomography image interpretation. The sagittal view of the right kidney demonstrates necrosis in the whole region and vacuoles in the renal cortex (E). The histological findings include numerous inflammatory cell infiltrates in the ureter and severe necrosis in the ureter and glomerulus $(\times 100)(\mathrm{F})$. were ligated with a size 0 silk suture (Black silk, AILEE Corp., Korea) and resected. Abdominal lavage was performed with sterile warm saline before closure. The purulent contents of the removed kidney and ureter were used for bacterial isolation and histological evaluation. Ureteral uroliths were analyzed for the composition of the stones. The resected kidney revealed that the renal pelvis and parenchyma were filled with copious brown pus. During hospitalization, Ampicillin $(22 \mathrm{mg} / \mathrm{kg}$ intravenous [IV] every $8 \mathrm{~h}$ [q8h], Penbrex inj.; Yungjin, Korea), Enrofloxacin $(5 \mathrm{mg} / \mathrm{kg}$ IV q24h, Baytril inj.; Byer Korea, Korea), Famotidine (0.5 $\mathrm{mg} / \mathrm{kg}$ IV q12h, Gaster inj.; Dong-A Pharm, Korea), Butorphanol $(0.01 \mathrm{mg} / \mathrm{kg}$ IV q8h, Buthophan inj.; Myungmoon Pharm, Korea), and Lactated ringer solution $(10 \mathrm{~mL} / \mathrm{kg} \mathrm{IV}$ q1h; CJ Health care, Korea) were administered. The patient recovered after the surgery without any complication and was discharged on postoperative day 3. E. coli was isolated from the pus, and it was sensitive to ampicillin and enrofloxacin. The stone was consisted of $80 \%$ calcium oxalate monohydrate and $20 \%$ magnesium ammonium phosphate (struvite). Histological findings confirmed severe pyelonephritis effacement of almost the entire renal architecture that could be significantly compromising renal function. The ureter had severe inflammation with focal ulceration. At the 6-month follow-up, the clinical signs had improved, and no complications were observed.

EPN is an uncommon condition in both humans and animals. The most common cause of EPN is diabetes mellitus with excretion of excess glucose in the urine and infection by gas-producing bacteria. Ureteral reflux induced by upper urinary tract obstruction could also cause EPN [3]. There are 2 
reports of gas formation in the urinary tract associated with diabetes mellitus in dogs whose prognosis was guarded because of emphysematous pyelitis and cystitis during the medical treatment $[5,6]$. In addition, in one dog with a congenital portosystemic shunt disease, ammonium urate ureteral stone caused ureteral obstruction and resulted in pyelonephrosis associated with E. coli infection [7]. Common bacterial causes of EPN include E. coli, Klebsiella species, Aerobacter spp., and Proteus species in humans $[2,3,8,9]$. In our case, E. coli was also isolated in the purulent exudate in the resected kidney.

This is the first report of type 1 EPN due to calcium oxalate uroliths of the upper urinary tract of a dog. Ureteral urine reflux to the pelvis might cause emphysematous infection of the kidney. In this case, pus and gas were localized in the renal parenchyma, pelvis, and ureter but not in the bladder. We suppose that although the dog was female, calcium oxalate uroliths had formed first because of the acidity of urine, and struvite was secondarily involved by urinary tract infection.

To diagnose EPN, ultrasonography had been commonly used in veterinary patients. In our case, multiple bright echogenicities associated with acoustic shadowing in the renal pelvis and ureter parts were seen, similar to human cases of EPN $[3,4,10]$. The dirty shadow artifact was characterized by a chaotic display of continuous echoes throughout the field. $\mathrm{CT}$ is the most specific diagnostic method for monitoring gas-containing urinary tract infections in humans $[4,6]$. CT is superior for excluding complex lesion of emphysematous pyelitis including renal fluid accumulation, abscesses, or EPN. In this report, we performed CT in an awake patient because of the poor body condition and the owner's hesitance to general anesthesia. This is the first time CT was performed in an awake dog to diagnose EPN. In our case, CT revealed gas contents in the renal parenchyma with little deterioration of image quality due to slight motion in the awake patient. Previous reports have also demonstrated CT in awake or sedated dogs with satisfactory image quality $[10,11]$. For the definitive diagnosis of upper airway obstruction on brachycephalic dogs, CT and 3-dimensional rendered endoscopy were performed without general anesthesia [10]. Another report demonstrated that contrast-enhanced multidetector CT was useful to detect pulmonary thromboembolism with pyothorax in an awake dog [12]. In this case, we did not performed dynamic CT using iodinated contrast media to prevent the renal injury. Moreover, we were worried about the excessive movement of the dog due to the burning sensation when contrast medium was injected. Therefore, we recommend CT in awake dogs that are obedient and have a high risk of anesthetic complications for the definite diagnosis of critical diseases that do not essentially need dynamic CT, such as EPN, laryngeal or tracheal airway obstruction, pulmonary thromboembolism, etc.

In our case, we performed ureteronephrectomy as a treatment option because histopathological findings showed that most of the renal medullary structures were destroyed because of severe inflammation. Preserving the damaged kidney using insertional ureteral stents or ureterotomy to remove only the ureteral stones have been attempted. However, the results show poor prognosis and high possibility of sepsis [13]. In the cat, a $2.5-\mathrm{Fr}, 14-\mathrm{cm}$ ureteral stent was inserted through the bladder and proximal ureter for the management of EPN; however, after 9 postoperative months, nephrectomy was performed because of the recurrence of hydronephrosis [13]. In humans, traditional ureteronephrectomy is also recommended in type $1 \mathrm{EPN}$, although ultrasound-guided percutaneous drainage catheters are used for the treatment of type 2 EPN $[1,9,14]$. Therefore, in cases of type 1 EPN with severe inflammation, aggressive surgical resection is appropriate for veterinary patients.

In conclusion, this was the first report of type 1 EPN associated with calcium oxalate uroliths. CT in the awake status was effective in reducing the risks of complications of general anesthesia and in evaluating the disease condition accurately. In addition, we recommend ureteronephrectomy in dogs with type 1 EPN when the contralateral kidney is intact in function for rapid recovery and good prognosis. Further studies are necessary to confirm the effectiveness of other non-surgical alternatives for dogs with type 2 EPN.

\section{Acknowledgements}

This work was supported by the Global Research and Development Center (GRDC) Program through the National Research Foundation of Korea (NRF) funded by the Ministry of Education, Science and Technology (2017K1A4A 3014959) and NRF-2018R1D1A1B07050014. This work was also supported by the research grant of the Chungbuk National University in 2017.

\section{References}

1. Pontin AR, Barnes RD. Current management of emphysematous pyelonephritis. 2009;6:272-279.

2. Ubee SS, McGlynn L, Fordham M. Emphysematous pyelonephritis. BJU Int 2011;107:1474-1478.

3. Huang JJ, Tseng CC. Emphysematous pyelonephritis: clinicoradiological classification, management, prognosis, and pathogenesis. Arch Intern Med 2000;160:797-805.

4. Kiris A, Ozdemir H, Bozgeyik Z, Kocakoc E. Ultrasonographic target appearance due to renal calculi containing gas in emphysematous pyelitis. Eur J Radiol Extra 2004;52:119-121.

5. Fabbi M, Manfredi S, Bianchi E, Gnudi G, Miduri F, Volta A. Emphysematous pyelitis and cystitis associated with vesicoureteral reflux in a diabetic dog. Can Vet J 2016;57: 382-386.

6. Moon R, Biller DS, Smee NM. Emphysematous cystitis and pyelonephritis in a nondiabetic dog and a diabetic cat. J Am Anim Hosp Assoc 2014;50:124-129.

7. Lim J, Yoon Y, Jung D, Yeon S, Lee H. Emphysematous pyonephrosis associated with extrahepatic portosystemic shunt in a dog. J Vet Med Sci 2016;78:697-700.

8. Kua C, Abdul Aziz Y. Air in the kidney: between emphyse- 
96 Ho-Hyun Kwak, Kamal Hany Hussein, Heung-Myong Woo, Kyung-Mee Park

matous pyelitis and pyelonephritis. Biomed Imaging Interv $\mathrm{J}$ 2008;4:e24.

9. Wong YN, Suh B, Parsons R, Telivala B, Haas N, Chen D, Greenberg R. Emphysematous pyelonephritis caused by Pasteurella multocida. Infect Med 2009;26:60.

10. Stadler K, Hartman S, Matheson J, O'Brien R. Computed tomographic imaging of dogs with primary laryngeal or tracheal airway obstruction. Vet Radiol Ultrasound 2011;52: 377-384.

11. Shanaman MM, Hartman SK, O'Brien RT. Feasibility for using dual-phase contrast-enhanced multi-detector helical computed tomography to evaluate awake and sedated dogs with acute abdominal signs. Vet Radiol Ultrasound 2012;53: 605-612.
12. Ngwenyama TR, Herring JM, O'Brien M, Hartman SK, Galloway KA, O'Brien RT. Contrast-enhanced multidetector computed tomography to diagnose pulmonary thromboembolism in an awake dog with pyothorax. J Vet Emerg Crit Care (San Antonio) 2014;24:731-738.

13. Gould EN, Cohen TA, Trivedi SR, Kim JY. Emphysematous pyelonephritis in a domestic shorthair cat. J Feline Med Surg 2016; 18:375-363.

14. Somani BK, Nabi G, Thorpe P, Hussey J, Cook J, N'Dow J; ABACUS Research Group. Is percutaneous drainage the new gold standard in the management of emphysematous pyelonephritis? Evidence from a systematic review. J Urol 2008; 179:1844-1849. 